\title{
R-GemOx Regimen
}

National Cancer Institute

\section{Source}

National Cancer Institute. R-GemOx Regimen. NCI Thesaurus. Code C140731.

A regimen consisting of rituximab, gemcitabine and oxaliplatin used in the treatment of diffuse large B-cell lymphoma (DLBCL). 